\title{
State of the art of robotic surgery related to vision: brain and eye applications of newly available devices
}

This article was published in the following Dove Press journal: Eye and Brain

\section{Raffaele Nuzzi \\ Luca Brusasco}

Department of Surgical Sciences, Eye Clinic, University of Torino, Turin, Italy
Correspondence: Luca Brusasco Department of Surgical Sciences, Eye Clinic, University of Torino, Corso Dogliotti 14, 10100, Turin, Italy Email luca.brusasco@yahoo.it
Background: Robot-assisted surgery has revolutionized many surgical subspecialties, mainly where procedures have to be performed in confined, difficult to visualize spaces. Despite advances in general surgery and neurosurgery, in vivo application of robotics to ocular surgery is still in its infancy, owing to the particular complexities of microsurgery. The use of robotic assistance and feedback guidance on surgical maneuvers could improve the technical performance of expert surgeons during the initial phase of the learning curve.

Evidence acquisition: We analyzed the advantages and disadvantages of surgical robots, as well as the present applications and future outlook of robotics in neurosurgery in brain areas related to vision and ophthalmology.

Discussion: Limitations to robotic assistance remain, that need to be overcome before it can be more widely applied in ocular surgery.

Conclusion: There is heightened interest in studies documenting computerized systems that filter out hand tremor and optimize speed of movement, control of force, and direction and range of movement. Further research is still needed to validate robot-assisted procedures.

Keywords: robotic surgery related to vision, robots, ophthalmological applications of robotics, eye and brain robots, eye robots

\section{Introduction}

Advances in tissue engineering and drug development continue to drive the need for innovative surgical techniques that can be performed in confined, difficult to visualize spaces and that allow the removal of small quantities of material from within the eye. Robots are a recent addition to the surgical team. The first surgical robots, Unimate PUMA 560 (Unimation, Danbury, CT, USA) and NeuroMate (Integrated Surgical Systems, Davis, CA, USA), were adaptations of late 1980s robotic technology from the industrial sector to neuronavigation in neurosurgery and stereotactic biopsy. ${ }^{1}$ The first true application of robots in medicine was in cardiosurgery, with a robot-assisted coronary bypass procedure. ${ }^{2}$

Since their introduction in cardiosurgery, robots have entered all surgical subspecialties. Hundreds of robotic systems are commercially available, and the most widely known are the da Vinci System (Intuitive Surgical, Sunnyvale, CA, USA), Zeus and Aesop (Computer Motion, Goleta, CA, USA), RoboDoc (Integrated Surgical Systems, Sacramento, CA, USA), and Naviot (Hitachi Ltd., Tokyo, Japan). ${ }^{3}$ Advanced robots now assist surgeons in procedures, which were unthinkable just a few years ago, ranging from minimally invasive surgery in laparoscopy to complex reconstruction surgery. 
Despite advances after the advent of phacoemulsification and femtosecond laser-assisted cataract surgery, in vivo application of robotics in ocular surgery is still in its infancy owing to the particular complexities of microsurgery in which high-precision microinstruments are rapidly manipulated. ${ }^{4,5}$ However, before near-perfect outcomes can be attained, many challenges remain to be met. One of the most technically demanding microsurgical procedures is vitreoretinal surgery, owing to the high precision in manipulating extremely delicate tissues within the confined space of the eye, often at forces below the threshold of tactile perception. The main technical limitations are inadequate spatial resolution and depth perception of microstructures for identifying tissue planes, imprecise maneuvers during micromanipulation of tissues due to natural hand tremor and lack of force, and dissection maneuvers below the surgeon's tactile perception threshold. Robotic surgery may provide the means to overcome these limitations. The use of robotic assistance and feedback guidance on surgical maneuvers could improve the technical performance of expert surgeons and novice surgeons during the initial phase of the learning curve. Unfortunately, however, many of the currently available robotic systems were not designed for ophthalmic applications, and so they have neither the mechanical resolution nor the flexibility of position required for intraocular procedures. The central design principle of modern instrumentation should ideally place the ophthalmic surgeon in complete control of every step of an operation.
Based on this principle, various robot projects have been tested on animal (eggs, chicken embryo, porcine eyes, etc.) and artificial material (phantom eyes specially designed to simulate robot systems) that mimic realistic situations of an eye disease.

\section{Potential advantages of surgical robots}

The extraordinary growth rate in the use of robot-assisted surgery is linked to its advantages over conventional surgical techniques. Filtration of hand tremor can reduce or eliminate the intrinsic human defect. Scaling of movement provides unprecedented precision otherwise impossible to achieve with unassisted manual techniques. Dexterity in confined anatomic spaces can be increased, as can maneuverability without direct visualization. Robots can also protect surgeons against hazardous exposure; telecontrol (telemedicine) of robotic systems can provide patients and surgeons expert in robotics with access to specialized procedures without the need to travel. Many of these advantages have already been realized. Figure 1 shows the da Vinci robot, the currently most widely used surgical platform.

The surgeon sits at a console where the surgical field is stereoscopically visualized. The manipulators and pedals control the instruments and the endoscopic camera. The computer translates the movements, scaling them and filtering and/or eliminating hand tremor $(156 \mu \mathrm{m}$ width $)$ in real time without any detectable delay. ${ }^{6}$

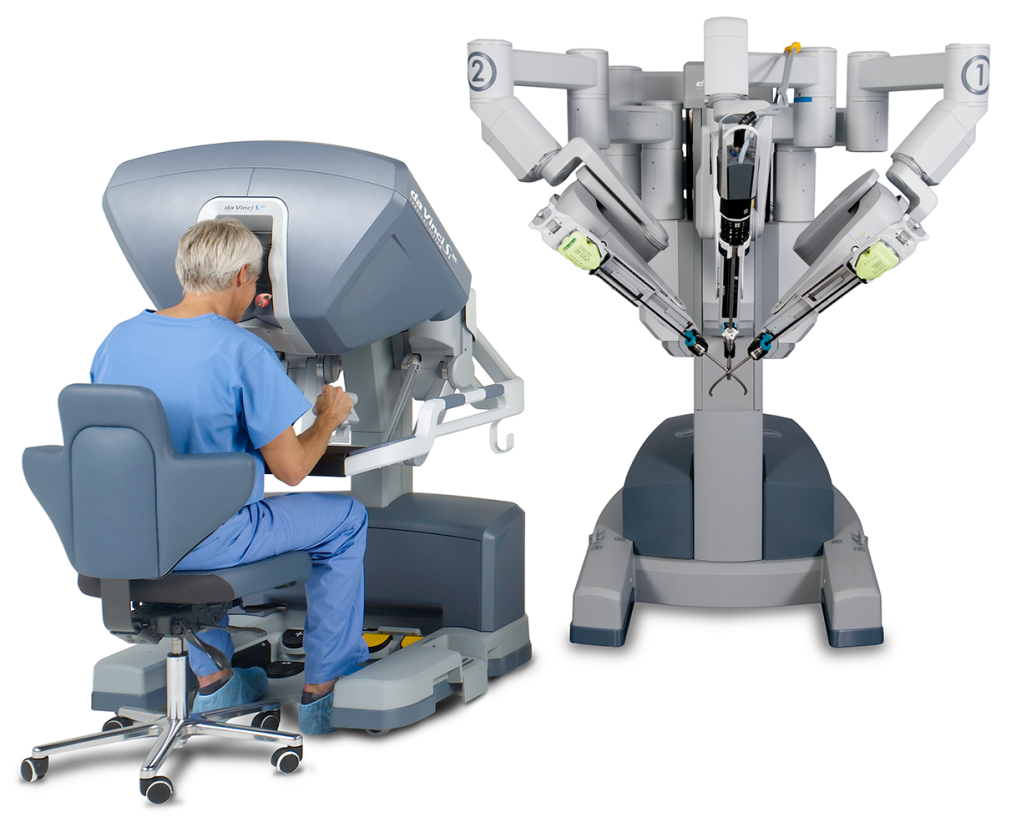

Figure I The da Vinci surgical robot.

Notes: Image courtesy of Intuitive Surgical Inc. da Vinci Si System with single-site instrumentation. Surgeon console, surgeon, da Vinci ${ }^{\circledR} \mathrm{Si}$ patient cart with Single-Site ${ }^{\mathrm{TM}}$ instruments. Available from: https://www.intuitivesurgical.com/company/media/images/singlesite.php. Accessed January 9, 20I7. Copyright @ 2018 Intuitive Surgical, Inc. ${ }^{66}$ 
Other commercially available robotic systems for special procedures include the Sensei X (Hansen Medical, Mountain View, CA, USA), which hemodynamic cardiologists use for cardiac catheter placement with great dexterity and without exposure to fluoroscopy, ${ }^{7}$ and the interactive robotic orthopedic system (MAKO Surgical Corp., Ft. Lauderdale, FL, USA), which increases surgical precision based on preoperative computed tomography scans. ${ }^{8}$

There are no commercially available robotic intraocular surgical platforms that incorporate the features of tested devices at competitive prices and with benefits superior to classical techniques. Ideally, a robotic surgical system is available at reasonable purchase and maintenance costs, is easy to use, and can be learned by novice surgeons. Such features have been validated individually for their ability to minimize injury from hand tremor, generate tactile feedback on depth and delicacy of the surgical procedures, and shorten operating time. It is not clear whether operating below the threshold of tactile sensitivity is necessary for achieving better outcomes. However, what is certain is that research must continue to find ways to increase the success rate of procedures such as robotic-assisted cannulation of occluded retinal veins.

\section{Disadvantages of surgical robots}

Despite the enormous potential and superiority of robotics reported in the literature, their use in the operating room is still limited by a number of drawbacks. The first is their high cost, for example, a da Vinci System costs over US\$1 million, to which annual maintenance and services costs of over US $\$ 100,000$ should be added. Operating room setup time may take longer and disrupt the surgical schedule; there are documented cases of da Vinci robot-assisted surgery requiring 20-30 minutes longer operating time. ${ }^{9}$ Ambulatory surgical procedures, which are common in ophthalmology and are becoming ever shorter, may not be amenable to robotic surgery, especially because operating time is an important outcome factor and because eye surgeons may doubt the advantage of adopting a robotic system in place of conventional procedures with low complications rates. Finally, besides the steep learning curve for new surgical applications, the complexity of surgical robots often poses a considerable challenge for clinicians and the surgical team in the operating room..$^{10}$

\section{Surgical robots related to vision: from neurosurgery to ocular surgery}

The first surgical robot was used in the 1980s to assist in achieving more accurate stereotactic biopsy in neurosurgery.
Since then, considerable steps forward have been made in central nervous system surgery and spinal surgery where advanced instruments provide technical assistance in instrument positioning, planning the trajectory, insertion of biopsy needles, implantation of brain stimulators, monitoring epilepsy using electrodes, and tissue resection. The neurosurgical robotic platforms are listed in a review published in 2014. ${ }^{47}$

Most of the recent surgical innovations in ophthalmology are derived from technological innovations. In anterior segment surgery, for example, excimer laser followed by femtosecond laser provide an accurate reconfiguration of the cornea, and new indications include automated capsulorhexis and lens softening/fragmenting before cataract extraction. In vitreoretinal surgery, micro-sized tools, high-speed cutters, and panoramic visualization systems have made significant progress. Can robot-assisted surgery lead to a revolution in the near future?

There are unique engineering challenges in robotic ophthalmic surgery. Extremely high precision is fundamental; for example, epiretinal membrane (ERM) peeling and other procedures require a tolerance on the order of microns, not millimeters. Many eye specialists have worked and continue to work with engineers to design and develop robotic systems that meet the needs of ophthalmic surgery.

Currently, there are no commercially available robotic systems that have been clinically tested for use in ophthalmic surgical procedures on human eyes. Research using artificial ocular systems or animal models has aimed to determine whether robotics from other surgical specialties can be applied or new systems developed.

\section{The da Vinci robot}

The da Vinci robotic system, widely used in diverse surgical applications, has led to a dramatic increase in the number of robot-assisted procedures in general surgery (e.g., thyroid, abdominal, gastroesophageal, and rectal surgery), urologic surgery (e.g., prostatectomy and retroperitoneal procedures), gynecological surgery (lower abdominal procedures), and head and neck surgery (transoral approaches in skull base procedures). An important application in neurosurgery is therapy for $\mathrm{C} 1-\mathrm{C} 2$ spinal disorders, anterior lumbar interbody fusion (ALIF), spinal vertebral surgery, ${ }^{57,58}$ and applications in neuro-oncology. ${ }^{46}$

The number of robot-assisted procedures increased from 1500 in 2000 to over 20,000 just 4 years later in 2004. In ophthalmology, robots have been used in anterior segment surgery to repair corneal laceration and to perform 
penetrating keratoplasty on human cadavers and porcine eyes, ${ }^{11}$ and on the posterior segment as well. Bourla et al ${ }^{12}$ achieved some success in extracting foreign bodies and performing capsulorhexis and $25-\mathrm{G}$ pars plana vitrectomy in porcine eyes.

The da Vinci system has six limitations in ocular surgery:

- A stable point of rotation above the robotic wrist renders ocular maneuvers less controllable.

- The endoscope-acquired images are inferior to those obtained with an ophthalmic microscope that permits direct visualization of the surgical field.

- High mechanical stress on eye structures (sclerostomy at instrument entry site) without control of the force applied. For this reason, Hexapod Surgical System (HSS) software was developed to improve surgical dexterity when using the da Vinci system. ${ }^{13}$

- Higher costs than conventional methods without demonstrable postoperative advantages.

- General anesthesia with neuromuscular block necessary also in outpatient procedures usually performed in local or topical anesthesia to prevent damage due to sudden patient movement, with increased operating time and costs, as well as health risks associated with general anesthesia.

- Less trust of patients and surgeons in new technologies.

\section{The intraocular robotic interventional surgical system (IRISS)}

The IRSS (Figure 2) is the result of combined efforts of the Jules Stein Eye Institute and the Department of Mechanical and Aerospace Engineering at University of California,
Los Angeles (UCLA) to provide a robotic platform for complete ophthalmic procedures. The system has a master controller and a slave manipulator; the controller comprises two joysticks, both of which are activated by the surgeon; the manipulator consists of two independent arms that can hold the surgical tools. Each mechanical arm has an independent wrist with 7 degrees of freedom to ensure significant freedom of movement for surgical maneuvers. Commercially available surgical instruments can be attached to the arms for specific maneuvers. ${ }^{14}$

The system is the first robotic platform for complete ocular procedures on the anterior and the posterior segment. It has been successfully used for capsulorhexis, removal of opaque lens cortex, central vitrectomy with detachment of posterior hyaloids, and simulation of temporal retinal vein microcannulation in porcine eyes. Each of the four procedures was successfully performed on four eyes without complications..$^{15,65}$

\section{The John Hopkins Steady-Hand Eye Robot}

This system (Figure 3) was designed to share surgical instrument control with the vitreoretinal surgeon. The mechanical system is composed of three main components:

- The XYZ system

- The roller mechanism

- The tilt system.

The XYZ system permits movement of the surgical instrument in all directions. The roller mechanism consists of a rotating table for optimizing access of the surgical instrument into the eye. The tilt mechanism is fixed to the surgical instrument holder at one end and to the roller

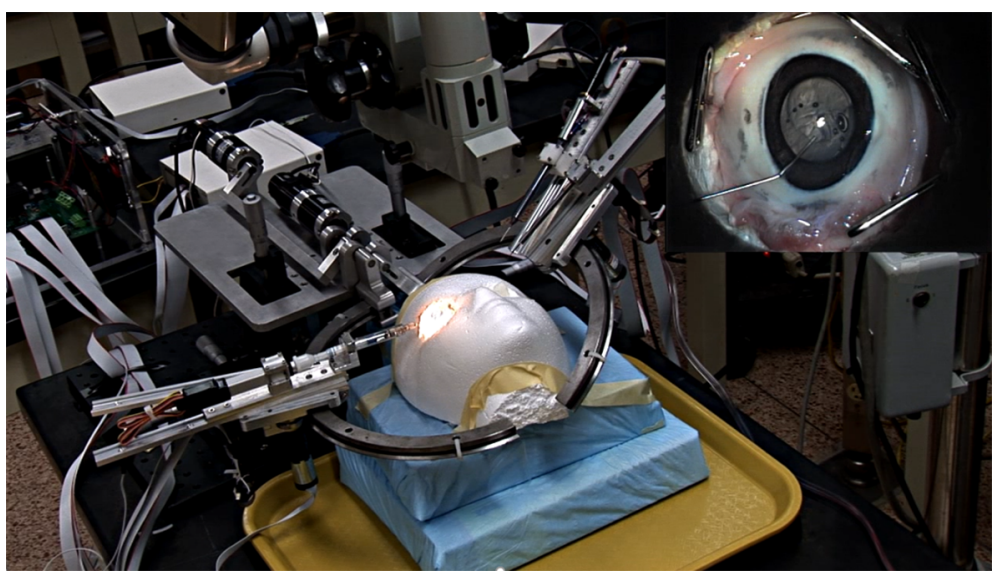

Figure 2 Intraocular robotic interventional surgical system.

Note: Image courtesy of UCLA.

Abbreviation: UCLA, University of California, Los Angeles. 


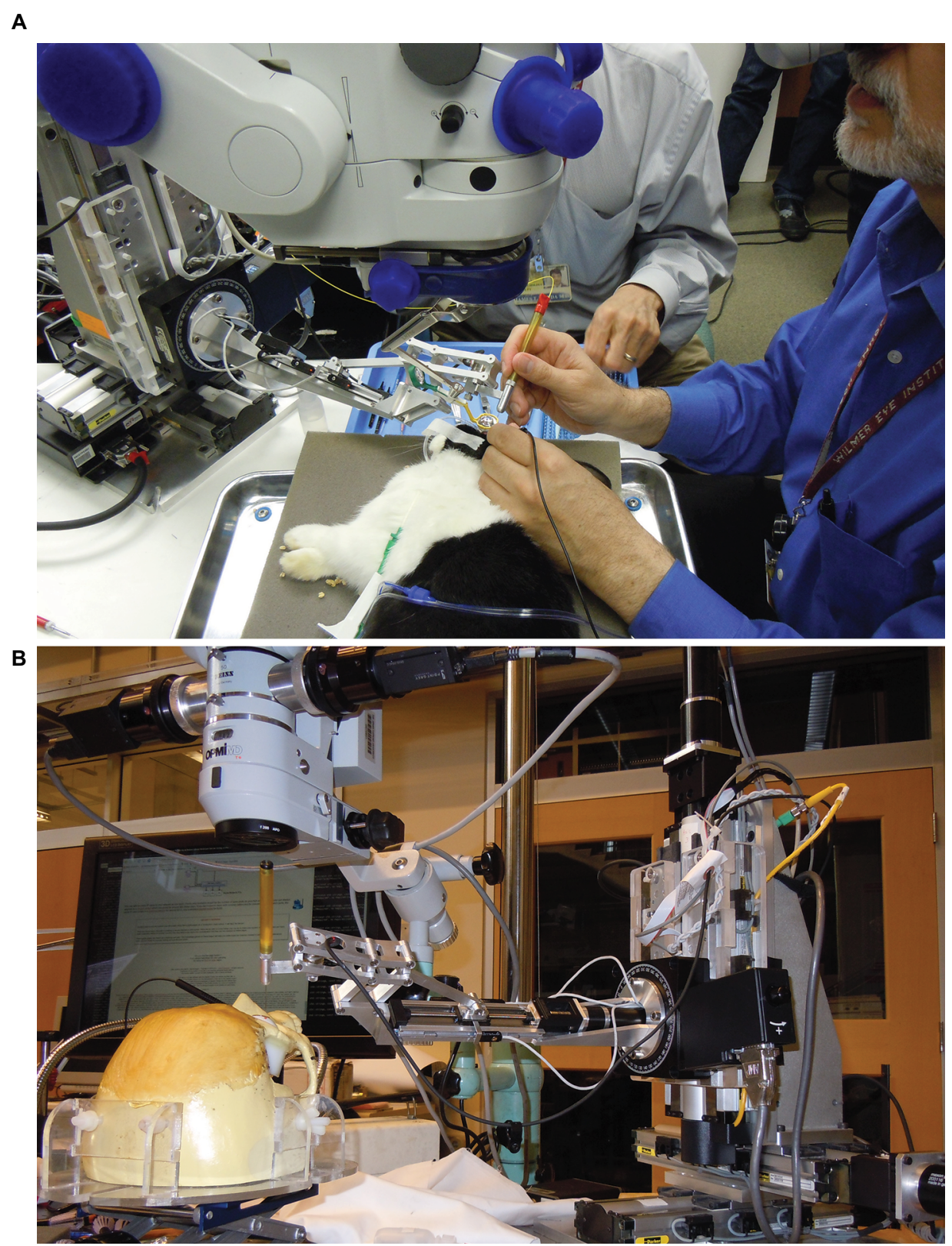

Figure 3 Experimental setup for testing JHU Steady-Hand Eye Robot with force-sensing tools: (A) during in vivo (with rabbits) and (B) dry eye phantoms experiments. Note: Images courtesy of John Hopkins University and Professor lordachita.

mechanism at the other end, allowing for varying degrees of angulation.

The roller mechanism is attached to a long tubular arm designed to separate the non-sterile parts of the robot from the sterile surgical field. Various conventional or modern intelligent surgical instruments can be attached to the tool holder. For example, a microforce sensor that provides feedback via audio signals can be used to guide the surgeon when manipulating the system. In this way, the robot improves movement to increase efficiency, whereas the microforce sensor guides the surgeon in applying optimal force during surgical action, enhancing the efficacy of each movement.

The team that created the platform also designed a force sensor with 3 degrees of freedom to measure the forces in all directions and for integration with the robot or used free hand. The tool holder has a rapid release mechanism with two release thresholds that allow the surgeon to quickly remove the instrument from the patient's eye during an unexpected event such as sudden head movement. ${ }^{16}$ Although research with this system has been limited to ocular microsurgery, possible benefit may also be gained in neurosurgery. ${ }^{56}$ 


\section{Smart instruments}

In the majority of systems designed for use in neurosurgery and ocular surgery, the most important parts are the end mechanical parts of the robotic platform. Accordingly, high expectations are placed on their practical performance. Independent of the form of the tool (rigid, straight, curved, etc.), freedom of movement is often the most important factor because there can be no margin of error when working within a confined space. Although these instruments have revolutionized surgical practice, many systems lack a human characteristic: proprioception. Proprioception refers to the ability to understand one's position through the aid of tactile feedback. Although this quality is essential for many surgical maneuvers, it has not been reproduced in any robotic system designed to date. Because excessive force can cause permanent iatrogenic damage during neurosurgical and ocular procedures, this factor must be taken into account in technological research. Wagner et $\mathrm{al}^{59}$ demonstrated that iatrogenic tissue damage is less with the use of robotic systems incorporating force feedback mechanisms.

Technical innovations will transform current surgical instruments into intelligent instruments that accompany the surgeon during each step of a surgical procedure. For example, intelligent forceps (Figure 4) were developed from conventional tools by fitting them with force sensors that measure the force applied to ocular tissue and communicate with the surgeon in real time via an acoustic feedback system. The system is able to detect microforces below the threshold of human tactile perception and sense when excessive force applied to tissues could cause intraoperative complications. ${ }^{17-63}$

Another example is the application of optical coherence tomography (OCT) to obtain scans in vivo during surgery. Tao et a ${ }^{18}$ demonstrated this concept in the operating room by coupling OCT with a common microscope. This system could aid the surgeon during epiretinal membrane peeling by enhancing visualization and improving the surgical outcome of complete delamination, as reported by Yang et al. ${ }^{19}$

Another intelligent instrument is the Micron, a microhandle designed to reduce hand tremor and improve the precision of passages. The handle detects movements, identifies hand tremor, and moves the tip so as to counteract involuntary movement. ${ }^{20}$ Hubschman et $\mathrm{al}^{21}$ described the use of a microhand for robot-assisted vitreoretinal surgery, a pneumatically driven microscopic surgical forceps for removing retinoic tissue from the retinal pigmented epithelium in porcine eyes, thus minimizing iatrogenic damage during delicate operator movements.

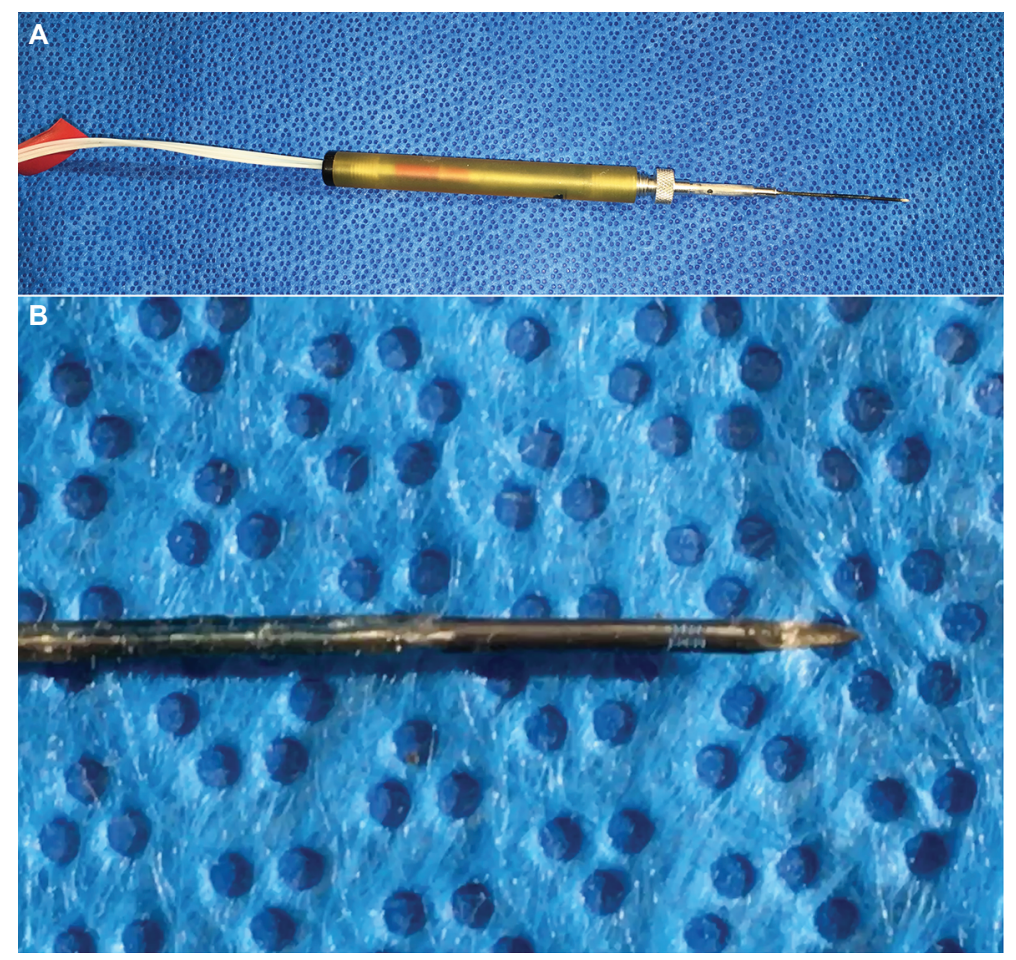

Figure 4 Tips of intelligent instruments incorporating force sensors.

Notes: (A) Photograph showing the tip of the instrument with 3 degrees of freedom. (B) Photograph of a handle with embedded forces sensors. Images courtesy of John Hopkins University and Professor lordachita. 


\section{Main applications of robotics in functional and stereotactic neurosurgery}

Image-guided robotic systems are ideal for functional applications, particularly for therapeutic procedures targeting difficult to access anatomic sites such as the globus pallidus, the ventral intermediate nucleus of the thalamus, and the subthalamic nucleus. For example, 113 deep brain stimulation electrodes were implanted using the NeuroMate platform, ${ }^{48}$ neuronavigation was carried out using an integrated platform system, the StimPilot (Medtronic Inc., Minneapolis, MN, USA), ${ }^{49}$ and other systems were used for deep electrode implantation in the treatment of drug-resistant epilepsy. ${ }^{50}$

The advantages of robotic stereotactic neurosurgery over conventional stereotactic neurosurgery have been addressed in terms of precision, ${ }^{54}$ setup time, and advancing along the learning curve. ${ }^{55}$ The evolution of stimulation device technology may lead to a different surgical approach and a wider application of robotics, particularly in multiple-target implantations and brainstem procedures. ${ }^{61-63}$ Although some research has been done on brain areas possibly related to vision, to best of our knowledge, only one study ${ }^{23}$ was published in 2017 on a robotic application in a brain area directly linked to vision: the sella turcica. This is the first clinical study regarding transoral robotic surgery (TORS) for sellar tumors that can compress the optic chiasm and result in bitemporal hemianopsia. This minimally invasive technique, performed using the da Vinci Surgical System, seems feasible as an innovative neurosurgical procedure that could minimize or obviate the adverse effects and disadvantages of the classical transsphenoidal route.

Finally, while spinal surgery is another potential area for the use of robotics in neurosurgery, its application has remained behind the substantial increase in minimally invasive spinal procedures. Recent retrospective and prospective studies comparing robotic surgery and conventional surgery have produced uneven results for robot-assisted open surgery. ${ }^{51-53}$

\section{Main areas of study in ophthalmology \\ Vitreoretinal surgery (ERM peeling)}

Vitreoretinal surgery poses three fundamental problems for surgeons: difficulty in estimating applied force and control of instrument position; suboptimal microscopic visualization; and physiological hand tremor. First, the surgeon must carefully position the instruments so as to avoid exerting excessive force on trocars entry sites and minimize the risk of iatrogenic damage to the eye. The second problem regards the difficulty in estimating the distance between the instrument tips and the target tissue under microscopy. Instruments are usually manipulated while observing the shadow they cast and estimating their distance; however, this maneuver is particularly challenging for novice surgeons. The third problem is that the precision of positioning in vitreoretinal surgery is $\sim 10 \mu \mathrm{m}$. Given that the mean amplitude of hand tremor is about $100-150 \mu \mathrm{m}$, only expert surgeons can manipulate instruments precisely and carry out a surgical procedure with good clinical results.

Informatics systems have been developed to aid the surgeon in performing microsurgical procedures. Mitchell et $\mathrm{al}^{22}$ developed a stability system consisting of a palm-held device through which the surgeon and the system cooperate in controlling the instrument via force sensors: the data are filtered to provide position control that is smooth and precise and with less force applied at the instrument tip.

One of the areas in vitreoretinal surgery where robotics has gained a place alongside conventional manual techniques is ERM peeling. Experimental studies have shown that robotic assistance could make performance more accurate. In 2013, Sunshine et al tested a microforce sensor embedded in a handpiece for measuring the forces generated during vitrectomy in rabbits and chorionic membrane peeling in chicken eggs. The results showed that minimal differences in forces exerted during normal maneuvers and forces slightly above the threshold were sufficient for creating complications. The use of a force sensor with audio feedback showed that the force required for ERM peeling was less than that needed for manual peeling. ${ }^{23}$ For this reason, many researchers believe in the potential of robotic assistance to improve procedure safety and standardization in eyes with macular pucker and in eyes with severe myopia differing in anatomy and extremely delicate retinas.

Systems coupled with OCT technologies can help the surgeon understand where to start peeling the membrane by identifying a larger space between the retina and the ERM. The use of this technique may eventually obviate the need for injecting indocyanine green dye, which is currently used to identify the internal limiting membrane and may be toxic for the retina.

During the annual meeting of the Association for Research in Vision and Ophthalmology (ARVO, Baltimore, MD, USA, 2017), the preliminary results of an experimental study were presented on the use of a real-time intraoperative spectral domain OCT (Rescan 700) integrated into an OPMI 
Lumera 700 microscope (both Carl Zeiss Meditec AG, Jena, Germany) and coupled with a robotic platform for localizing instruments within porcine eyes. ${ }^{24}$ Conventionally, surgeons use stereopsis and the shadow cast by the instruments on the retina to guide them. In this study, the innovative OCT technology provided visualization of the retinal microanatomy in real time, enhanced precision in controlling instrument depth inside the eye in relationship to its anatomical structures, and improved accuracy on the order of $10 \mu \mathrm{m}$. A future clinical trial with this technique is planned using subretinal injection of tissue plasminogen activator (tPA) for submacular hemorrhages and stem cells in patients with age-related macular degeneration.

\section{Vessel cannulation and release of intravascular drugs}

Vessel cannulation is necessary in the treatment of vascular disorders such as retinal vein occlusion, arteriovenous malformation, and retinal microaneurysm. The procedure is more difficult to perform peripherally than in the areas near the optic nerve due to the difference in vessel lumen diameter and the lack of anchorage to underlying structures as is the case at the edge of the optic nerve. Vessels are also larger near the optic nerve and rapidly decrease in size in peripheral tissues.

Robotic assistance in technically demanding procedures like microcannulation of retinal veins may be warranted because of the small diameter of the veins and physiological hand tremor. Robotic assistance might filter out tremor and also help guide surgical movement to positioning a microcannula in the retinal blood vessel and maintain its position for several minutes during which drugs are injected to dissolve the thrombi that have formed..$^{25,26}$ Thus, robotic assistance could provide a theoretical advantage of enhanced dexterity and precision, in addition to incorporating a new technology that could translate into improved clinical results. Microcannulation in vessels measuring $80 \mu \mathrm{m}$ in diameter has been successfully demonstrated in chicken embryos.

Ueta et $\mathrm{al}^{27}$ first described in 2009, and then later reported in $2011^{28}$ on a system for microvessel cannulation of porcine eyes. Comparison of robot-assisted and manual procedures carried out by two expert surgeons showed greater precision in the initial stage of cannulation and particularly in the second stage of maintaining the tip inside the vessel lumen long enough for the drug release. The robot helps to maintain the tip inside the vessel because there is no human tremor effect. On the other hand, in the manual procedure, this is impossible because the amplitude of the normal hand tremor of the surgeon (even if this was experienced by an expert surgeon) is greater than the vessel diameter and the tip would exit easily from the lumen if a manual procedure is attempted.

In a more recent study published in 2016 , Gijbels et $\mathrm{al}^{29}$ reported successful intravascular drug release in 20 of 25 porcine eyes with the use of robot assistance by a young surgeon. de Smet et $\mathrm{al}^{30}$ published the results of their study in which 5- to 7-week-old piglets were anesthetized and endolaserinduced retinal venous thrombosis was created before vascular cannulation. Vascular occlusion was visualized via OCT and fluorangiography. By using the robotic system (Preceyes micromanipulator, Preceyes b.v., Endhoven, The Netherlands; Figure 5), the researchers were able to cannulate a vessel and release a balanced saline solution that revealed the occlusion.

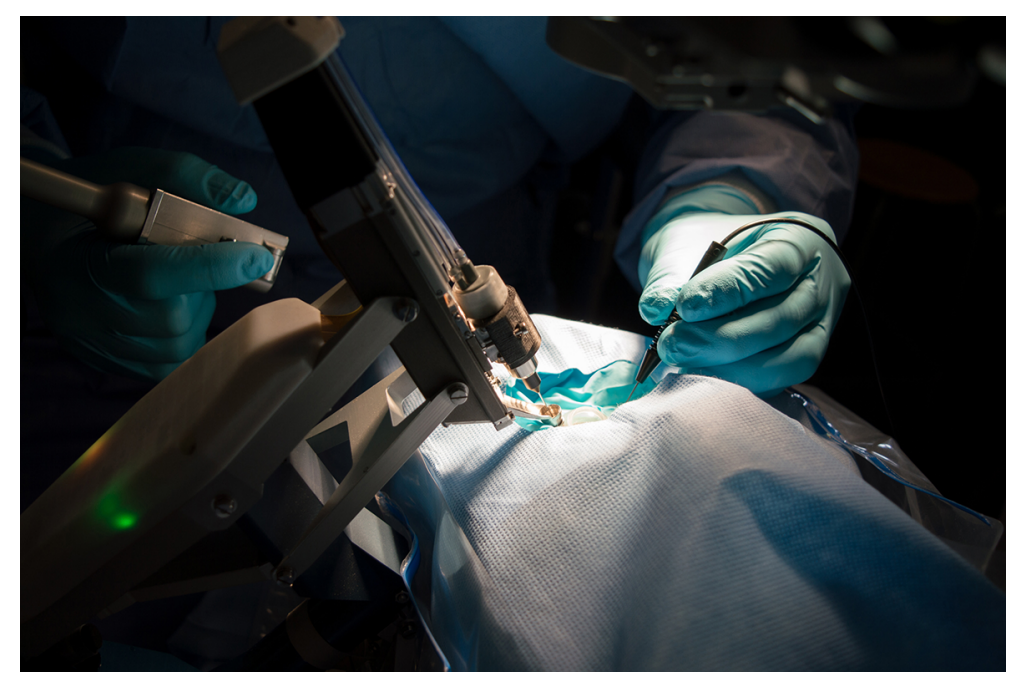

Figure 5 Preceyes micromanipulator.

Note: Image courtesy of Preceyes b.v., Endhoven, The Netherlands. 
The cannulation was successful for several minutes, being extended in their study up to 20 minutes. Saline was not able to release the occlusion, but using a plasmin derivate was successful with an injection of a few minutes. This suggested that the procedure may be performed in human eyes at a force below human tactile perception. ${ }^{64}$ One very recent report published in 2017 described refinements to the IRISS, highlighting the enhanced dexterity and precision the system allows in cataract surgery and retinal vein cannulation in porcine eyes, as assessed by intraoperative OCT. ${ }^{65}$

\section{First in vivo application of robot-assisted eye surgery}

Although much engineering effort has been dedicated to the development of robotic assistance in vitreoretinal surgery, the first in vivo application of robotic assistance in ophthalmology was an operation for pterygium in 2015. The entire procedure was carried out on a da Vinci SI HD platform (Intuitive Surgical) and reported by Bourcier et al. ${ }^{31}$ Although robotic assistance provided the dexterity necessary for executing the delicate manipulation of pterygium excision and conjunctival autografting, no improvement was observed in clinical practice between the robot-assisted and the manual procedure.

\section{Application of robotics as an aid in ocular laser surgery}

Recent progress in robotic assistance in minimally invasive laser surgery marked a step forward in increasing the precision, reproducibility, and simple automated maneuvers in surgery for glaucoma, cataracts, and corneal transplant. Becker et $\mathrm{al}^{32}$ integrated a robotic assistance system with laser technology for retinal photocoagulation and reported an increase in efficiency and a reduction in error. Yang et $\mathrm{al}^{33}$ tested the possible application of automated photocoagulation in artificial models. Two vitreoretinal surgeons performed the same procedure manually and with a manipulator attached to an endolaser. In the latter case, the manipulator corrected the errors between the real target and the laser beam directed by the operator.

A robotic manipulator for laser tissue repair of the sclera was tested by Garcia et al. ${ }^{34}$ Belyea et $\mathrm{al}^{35}$ performed transcleral cyclophotocoagulation of the ciliary bodies using a Telepresence Surgery System (TeSS) robot. The Espresso platform ${ }^{36}$ was developed for minor invasive laser surgery with the intent to personalize treatment. The contact force exerted by a device to increase ocular stability of the surgical instrument was directly related to intraocular pressure when preoperative anatomic characteristics are acquired, as typically occurs during tomometry. Yang et $\mathrm{al}^{37}$ reported better results with the Micron Robot System for retinal laser photocoagulation than with the manual procedure. The Micron Robot System comprises the handheld manipulator, an optical tracking system, and a real-time controller. The manipulator incorporates a miniature Gough-Stewart platform actuated by six ultrasonic linear motors (SQUIGGLE ${ }^{\circledR}$ SQL-RV-1.8; New Scale Technologies, Inc., Victor, NY, USA). The custom-built optical tracking system ("Apparatus to Sense Accuracy of Position" or "ASAP") provides the position and orientation at a sampling rate of $1 \mathrm{kHz}$ over a $27 \mathrm{~cm}^{3}$ workspace, with less than $10 \mu \mathrm{m}$ RMS noise. The vision system delivers visual feedback to the Micron controller, such as the locations of the laser tip and aiming beam, the 3D surface of a target, and the tracking of the surface. The system consists of a stereomicroscope (Zeiss OPMI ${ }^{\circledR}$; Carl Zeiss Meditec AG) with variable magnification $(4-25 \times)$, two CCD cameras (Flea ${ }^{\circledR} 2$; Point Grey Research, Richmond, BC, Canada), and a desktop PC.

\section{Future outlook \\ Telerobotics and ocular cell therapy}

It is difficult to imagine where technological advances may lead; however, two future areas of focus for robotics are telemedicine and retinal implantation of stem cells. By coupling telecommunications technologies and robotic assistance, telerobotics could permit remote control of a platform by operators thousands of kilometers away from their patients. In this way, hand movement and vision are transformed into electrodigital signals, as done in teletraining in medical education and remote specialist exams. ${ }^{38-40}$

Robotic assistance could also benefit cell therapy and regenerative stem cell therapy in ophthalmology. Stem cells possess properties that can be harnessed for the treatment of systemic and eye diseases: they generate progenic cells that directly repair damage, that are integrated into pathological tissue, and that serve as gene therapy vectors. Studies have reported on subretinal transplantation of autologous iris pigment epithelium in the treatment of age-related macular degeneration. Thumann et $\mathrm{al}^{41}$ and Lappas et $\mathrm{al}^{42}$ reported on combined macular surgery and iris pigment epithelial cell translocation, with improvement or stabilization of vision in $90 \%$ of cases. Iris pigment epithelial cells are amenable to transplantation, easy to harvest, and similar to retinal pigment epithelial cells. In these and later studies, no rejection reactions were observed. Such cells have also been used as vectors for the release of target molecules ${ }^{43,44}$ and 
successfully implanted into the vitreous accumulate at the optic nerve head, suggesting their potential in cell therapy for neurodegenerative diseases such as glaucoma. Their potential in gene therapy will depend on whether they can produce neurotrophic factors for use by the optic nerve and for the evaluation of other cells, including mesenchymal, embryonal, neural, corneal limbar, adipocyte precursors, and Schwann cells. ${ }^{45}$

Robotic surgery could play a key role in cell regeneration, particularly during delicate intraocular procedures to introduce them into the intraretinal or subretinal space, because the risk of iatrogenic injury due to hand tremor or poor visualization would be devastating. In addition to these novel areas, other possible applications are nanotechnologies including nanorobots and nanodevices that can be introduced into the vascular system or anatomic cavities such as the eye or skull. A review listed the possible therapeutic possibilities of this technology in neurosurgery. ${ }^{60}$

\section{Conclusion}

Robot-assisted surgery has revolutionized many surgical subspecialties. Despite the giant steps forward made in neurosurgery over the past 20 years, a major obstacle remains the difficulty of today's platforms to provide tactile perception feedback when force is applied to a tissue, which could generate different resistance patterns depending on its physiochemical characteristics. Overcoming this limitation is particularly important for ocular surgery.

Since eye surgery is commonly performed under topical or local anesthesia, sudden voluntary or involuntary movement may result in iatrogenic damage to the patient unless the platform is equipped with a safety release mechanism the surgeon can activate. In manually controlled vitreoretinal surgical procedures, the surgeon can quickly remove the surgical instruments from the scleral entry points should the patient move suddenly. Such safety features would need to be incorporated in the design of robotic platforms.

Conventional microsurgery of the eye is carried out under direct visualization of anatomical structures via optic microscopy. While the anterior segment can be visualized directly, the posterior segment, with the retina and vitreous, is visualized via special lenses and visualization systems.

Robots could provide a different way to visualize the retina in $3 \mathrm{D}$ and through ultrathin transverse slices by coupling it with today's microscope technologies such as OCT.

Neither commercially available platforms nor systems currently under development have been able to combine the theoretical advantages of robot-assisted procedures in a single device. Many systems have not yet been validated in vivo. While recent studies have underlined the strengths and weaknesses of the systems tested so far, it will take clinical trials to demonstrate their potential in vivo. The use of a platform like the da Vinci System, jointly shared by several hospital services, for pterygium surgery in vivo, ${ }^{31}$ is a remarkable example of resource sharing among different users. However, this type of robotic surgery, like others, necessitates longer operating time and higher equipment purchase and maintenance costs than conventional pterygium surgery, without any real advantage for the patient.

Current limitations notwithstanding, we believe that robot-assisted eye surgery will expand therapeutic options, reduce complication rates, and continue to redefine procedures for treating clinical conditions that are still incurable today. There are numerous studies documenting computerized systems that filter out hand tremor and optimize speed of movement, control of force, and direction and range of movement. In addition, tissue physiological and chemical data can be detected by sensors embedded in the instrument tip so as to collect direct and indirect signals of tissue stress. Further research is needed to validate robotassisted procedures. Once standardized such procedures may be tested in humans.

\section{Disclosure}

This study was conducted without the support of any commercial or financial relationships that could be construed as a potential conflict of interest. The authors report no conflicts of interest in this work.

\section{References}

1. Moran ME. Evolution of robotic arms. J Robot Surg. 2007;1(2):103-111.

2. Cannon JW, Howe RD, Dupont PE, Triedman JK, Marx GR, del Nido PJ. Application of robotics in congenital cardiac surgery. Semin Thorac Cardiovasc Surg Pediatr Card Surg Annu. 2003;6:72-83.

3. Cleary K, Nguyen C. State of the art in surgical robotics: clinical applications and technology challenges. Comput Aided Surg. 2001;6:312-328.

4. Oleynikov D. Robotic surgery. Surg Clin North Am. 2008;88: 1121-1130,viii.

5. Fine HF, Wei W, Goldman R, Simaan N. Robot-assisted ophthalmic surgery. Can J Ophthalmol. 2010;45(6):581-584.

6. Iranmanesh P, Morel P, Wagner OJ, Inan I, Pugin F, Hagen ME. Set-up and docking of the da Vinci surgical system: prospective analysis of initial experience. Int J Med Robot. 2010;6(1):57-60.

7. Saliba W, Reddy VY, Wazni O, et al. Atrial fibrillation ablation using a robotic catheter remote control system: initial human experience and long-term follow-up results. J Am Coll Cardiol. 2008;51(25):2407-2411.

8. Pearle AD, Kendoff D, Stueber V, Musahl V, Repicci JA. Perioperative management of unicompartmental knee arthroplasty using the MAKO robotic arm system (MAKOplasty). Am JOrthop. 2009;38(2 suppl):16-19.

9. Salman M, Bell T, Martin J, Bhuva K, Grim R, Ahuja V. Use, cost, complications and mortality of robotic versus nonrobotic general surgery procedures based on a nationwide database. Am Surg. 2013;79(6):553-560. 
10. Lanfranco AR, Castellanos AE, Desai JP, Meyers WC. Robotic surgery: a current perspective. Ann Surg. 2004;239(1):14.

11. Tsirbas A, Mango C, Dutson E. Robotic ocular surgery. $B r J$ Ophthalmol. 2007;91(1):18-21.

12. Bourla DH, Hubschman JP, Culjat M, Tsirbas A, Gupta A, Schwartz SD. Feasibility study of intraocular robotic surgery with the da Vinci surgical system. Retina. 2008;28(1):154-158.

13. Bourges JL, Hubschman JP, Wilson J, Prince S, Tsao TC, Schwartz $\mathrm{S}$. Assessment of a hexapod surgical system for robotic micro-macro manipulations in ocular surgery. Ophthalmic Res. 2011;46(1):25-30.

14. He X, van Geirt V, Gehlbach P, Taylor R, Iordachita I. IRIS: integrated robotic intraocular snake. IEEE Int Conf Robot Autom. 2015;2015: 1764-1769.

15. Rahimy E, Wilson J, Tsao TC, Schwartz S, Hubschman JP. Robotassisted intraocular surgery: development of the IRISS and feasibility studies in an animal model. Eye (Lond). 2013;27(8):972-978.

16. Gonenc B, Handa J, Gehlbach P, Taylor RH, Iordachita I. A comparative study for robot assisted vitreoretinal surgery: micron vs. the steady-hand robot. IEEE Int Conf Robot Autom. 2013:4832-4837.

17. Cutler N, Balicki M, Finkelstein M, et al. Auditory force feedback substitution improves surgical precision during simulated ophthalmic surgery. Invest Ophthalmol Vis Sci. 2013;54(2):1316-1324.

18. Tao YK, Ehlers JP, Toth CA, Izatt JA. Intraoperative spectral domain optical coherence tomography for vitreoretinal surgery. Opt Lett. 2010;35(20):3315-3317.

19. Yang S, Balicki M, Wells TS, et al. Improvement of optical coherence tomography using active handheld micromanipulator in vitreoretina surgery. Conf Proc IEEE Eng Med Biol Soc. 2013;2013:5674-5677.

20. Maclachlan RA, Becker BC, Tabarés JC, Podnar GW, Lobes LA Jr, Riviere CN. Micron: an actively stabilized handheld tool for microsurgery. IEEE Trans Robot. 2012;28(1):195-212.

21. Hubschman JP, Bourges JL, Choi W, et al. 'The Microhand': a new concept of micro-forceps for ocular robotic surgery. Eye (Lond) 2010;24(2):364-367.

22. Mitchell B, Koo J, Iordachita I, et al. Development and application of a new steady-hand manipulator for retinal surgery. In: Proceedings 2007 IEEE International Conference on Robotics and Automation: 623-629. Available at: http://ieeexplore.iee.org/document/4209160/. Accessed January 12, 2018.

23. Sunshine S, Balicki M, He X, et al. A force-sensing microsurgical instrument that detects forces below human tactile sensation. Retina. 2013;33(1):200-206

24. Nasseri MA, Eslami A, Zapp DM, et al. Intraoperative OCT guided robotic sub-retinal surgery. ARVO Conference 2017. Baltimore, USA; 2017.

25. Nasseri MA, Eder M, Nair S, et al. The introduction of a new robot for assistance in ophthalmic surgery. Conf Proc IEEE Eng Med Biol Soc. 2013;2013:5682-5685.

26. Tanaka S, Harada K, Ida Y, et al. Quantitative assessment of manual and robotic microcannulation for eye surgery using new eye model. Int J Med Robot. 2015;11(2):210-217.

27. Ueta T, Yamaguchi Y, Shirakawa Y, et al. Robot-assisted vitreoretinal surgery. Development of a prototype and feasibility studies in an animal model. Ophthalmology. 2009;116(8):1538-43, 1543.e1-2.

28. Ueta T, Nakano T, Ida Y, Sugita N, Mitsuishi M, Tamaki Y. Comparison of robot-assisted and manual retinal vessel microcannulation in an animal model. Br J Ophthalmol. 2011;95(5):731-734.

29. Gijbels A, Willekens K, Esteveny L, Stalmans P, Reynaerts D, Vander Poorten EB. Towards a clinically applicable robotic assistance system for retinal vein cannulation. In: 2016 6th IEEE International Conference on Biomedical Robotics and Biomechatronics (BioRob). Available at: http:// ieeexplore.ieee.org/document/7523639/. Accessed January 12, 2018.

30. de Smet MD, Meenink TC, Janssens T, et al. Robotic assisted cannulation of occluded retinal veins. PLoS One. 2016;11(9):e0162037.

31. Bourcier T, Chammas J, Becmeur PH, et al. Robotically assisted pterygium surgery: first human case. Cornea. 2015;34(10):1329-1330.
32. Becker BC, MacLachlan RA, Lobes LA, Riviere CN. Semiautomated intraocular laser surgery using handheld instruments. Lasers Surg Med. 2010;42(3):264-273.

33. Yang S, Lobes LA Jr, Martel JN, Riviere CN. Handheldautomated microsurgical instrumentation for intraocular laser surgery. Lasers Surg Med. 2015;47(8):658-668.

34. Garcia P, Mines MJ, Bower KS, et al. Robotic laser tissue welding of sclera using chitosan films. Lasers Surg Med. 2009;41(1):59-67.

35. Belyea DA, Mines MJ, Yao WJ, Dan JA, Bower KS. Telerobotic contact transscleral cyclophotocoagulation of the ciliary body with the diode laser. J Robot Surg. 2014;8(1):48-55.

36. Russo S, Petroni G, Quaglia C, et al. ESPRESSO: a novel device for laser-assisted surgery of the anterior eye segment. Minim Invasive Ther Allied Technol. 2016;25(2):70-78.

37. Yang S, MacLachlan RA, Martel JN, Lobes LA Jr, Riviere CN. Comparative evaluation of handheld robot-aided intraocular laser surgery. IEEE Trans Robot. 2016;32:246-251.

38. Mylonas GP, Darzi A, Yang GZ. Gaze-contingent control for minimally invasive robotic surgery. Comput Aided Surg. 2006;11(5):256-266.

39. Jeganathan VS, Shah S. Robotic technology in ophthalmic surgery. Curr Opin Ophthalmol. 2010;21(1):75-80.

40. Rentschler ME, Platt SR, Berg K, Dumpert J, Oleynikov D, Farritor SM. Miniature in vivo robots for remote and harsh environments. IEEE Trans Inf Technol Biomed. 2008;12:66-75.

41. Thumann G, Aisenbrey S, Schraermeyer U, et al. Transplantation of autologous iris pigment epithelium after removal of choroidal neovascular membranes. Arch Ophthalmol. 2000;118(10): $1350-1355$.

42. Lappas A, Weinberger AW, Foerster AM, Kube T, Rezai KA, Kirchhof B. Iris pigment epithelial cell translocation in exudative age-related macular degeneration. A pilot study in patients. Graefes Arch Clin Exp Ophthalmol. 2000;238(8):631-641.

43. Abe T, Yoshida M, Yoshioka Y, et al. Iris pigment epithelial cell transplantation for degenerative retinal diseases. Prog Retin Eye Res. 2007;26(3):302-321.

44. Wert KJ, Skeie JM, Davis RJ, Tsang SH, Mahajan VB. Subretinal injection of gene therapy vectors and stem cells in the perinatal mouse eye. J Vis Exp. 2012;(69).

45. Jordan JF, Semkova I, Kociok N, Welsandt GR, Krieglstein GK, Schraermeyer U. Iris pigment epithelial cells transplanted into the vitreous accumulate at the optic nerve head. Graefes Arch Clin Exp Ophthalmol. 2002;240(5):403-407.

46. Onen M, Naderi S. Robotic systems in spine surgery. Turk Neurosurg. 2014;24(3):305-311.

47. Mattei TA, Rodriguez AH, Sambhara D, Mendel E. Current state-ofthe-art and future perspectives of robotic technology in neurosurgery. Neurosurg Rev. 2014;37(3):357-366. discussion 366.

48. Varma TR, Eldridge P. Use of the NeuroMate stereotactic robot in a frameless mode for functional neurosurgery. Int J Med Robot. 2006;2(2): $107-113$.

49. Heuer GG, Zaghloul KA, Jaggi JL, Baltuch GH. Use of an integrated platform system in the placement of deep brain stimulators. Neurosurgery. 2008;62(3 suppl 1):245-247. discussion 247-8.

50. Eljamel MS. Robotic neurological surgery applications: accuracy and consistency or pure fantasy? Stereotact Funct Neurosurg. 2009;87(2): 88-93.

51. Devito DP, Kaplan L, Dietl R, et al. Clinical acceptance and accuracy assessment of spinal implants guided with SpineAssist surgical robot: retrospective study. Spine (Phila Pa 1976). 2010;35(24):2109-2115.

52. Kantelhardt SR, Martinez R, Baerwinkel S, Burger R, Giese A, Rohde V. Perioperative course and accuracy of screw positioning in conventional, open robotic-guided and percutaneous robotic-guided, pedicle screw placement. Eur Spine J. 2011;20(6):860-868.

53. Schizas C, Thein E, Kwiatkowski B, Kulik G. Pedicle screw insertion: robotic assistance versus conventional C-arm fluoroscopy. Acta Orthop Belg. 2012;78(2):240-245. 
54. Kwoh YS, Hou J, Jonckheere EA, Hayati S. A robot with improved absolute positioning accuracy for CT guided stereotactic brain surgery. IEEE Trans Biomed Eng. 1988;35(2):153-160.

55. Bekelis K, Radwan TA, Desai A, Roberts DW. Frameless robotically targeted stereotactic brain biopsy: feasibility, diagnostic yield, and safety. J Neurosurg. 2012;116(5):1002-1006.

56. Uneri A, Balicki MA, Handa J, Gehlbach P, Taylor RH, Iordachita I. New steady-hand eye robot with micro-force sensing for vitreoretinal surgery. Proc IEEE RAS EMBS Int Conf Biomed Robot Biomechatron. 2010;2010(26-29):814-819.

57. Ponnusamy K, Chewning S, Mohr C. Robotic approaches to the posterior spine. Spine (Phila Pa 1976). 2009;34(19):2104-2109.

58. Wang T, Luan S, Hu L, Liu Z, Li W, Jiang L. Force-based control of a compact spinal milling robot. Int J Med Robot. 2010;6(2):178-185.

59. Wagner CR, Stylopoulos N, Howe RD. The role of force feedback in surgery: analysis of blunt dissection. Paper presented at: 10th Symposium on Haptic Interfaces for Virtual Environment and Teleoperator Systems; 2002; Orlando, FL.

60. Khawaja AM. The legacy of nanotechnology: revolution and prospects in neurosurgery. Int J Surg. 2011;9(8):608-614.
61. Mazzone P, Della Marca G, Sposato S, Di lazzaro V, Scarnati E. Tridimensional modelling of midbrain and pontine structures: a proposed approach to the stereotactic targeting of nucleus pedunculopontine tegmenti. Neurotarget. 2008;3:8-20.

62. Mazzone P, Scarnati E. Deep brain stimulation of the medial thalamus for movement disorders: the role of centromedian-parafascicular complex. In: Krames ES, Peckman PH, Rezai AR, editors. Neuromodulation. New York: Academic Press; 2009:599-615.

63. De Lorenzo D, De Momi E, Dyagilev I, et al. Force feedback in a piezoelectric linear actuator for neurosurgery. Int J Med Robot. 2011;7:268-275.

64. Molaei A, de Smet MD, Safi S, et al. Toward the Art of Robotic-assisted Vitreoretinal Surgery. J Ophthalmic Vis Res. 2017;12(2):212-218.

65. Wilson JT, Gerber MJ, Prince SW, et al. Intraocular robotic interventional surgical system (IRISS): mechanical design, evaluation, and master-slave manipulation. Int J Med Robot. Epub 2017 Jul 31.

66. Intuitive Surgical, Inc. da Vinci Si System with single-site instrumentation. Surgeon console, surgeon, da Vinci ${ }^{\circledR}$ Si patient cart with SingleSite ${ }^{\mathrm{TM}}$ instruments. Available from: https:/www.intuitivesurgical.com/ company/media/images/singlesite.php. Accessed January 9, 2017.
Eye and Brain

\section{Publish your work in this journal}

Eye and Brain is an international, peer-reviewed, open access journal focusing on clinical and experimental research in the field of neuroophthalmology. All aspects of patient care are addressed within the journal as well as basic research. Papers covering original research, basic science, clinical and epidemiological studies, reviews and evaluations,

Submit your manuscript here: https://www.dovepress.com/eye-and-brain-journal
Dovepress

guidelines, expert opinion and commentary, case reports and extended reports are welcome. The manuscript management system is completely online and includes a very quick and fair peer-review system, which is all easy to use. Visit http://www.dovepress.com/testimonials.php to read real quotes from published authors. 